This is an electronic reprint of the original article. This reprint may differ from the original in pagination and typographic detail.

Author(s): Kainulainen, Kimmo; Tuominen, Kimmo; Vaskonen, Ville

Title: Self-interacting dark matter and cosmology of a light scalar mediator

Year: $\quad 2016$

Version:

Please cite the original version:

Kainulainen, K., Tuominen, K., \& Vaskonen, V. (2016). Self-interacting dark matter and cosmology of a light scalar mediator. Physical Review D, 93(1), Article 015016. https://doi.org/10.1103/PhysRevD.93.015016

All material supplied via JYX is protected by copyright and other intellectual property rights, and duplication or sale of all or part of any of the repository collections is not permitted, except that material may be duplicated by you for your research use or educational purposes in electronic or print form. You must obtain permission for any other use. Electronic or print copies may not be offered, whether for sale or otherwise to anyone who is not an authorised user. 


\title{
Self-interacting dark matter and cosmology of a light scalar mediator
}

\author{
Kimmo Kainulainen, ${ }^{1,3, *}$ Kimmo Tuominen, ${ }^{2,3, \dagger}$ and Ville Vaskonen ${ }^{1,3,}$ \\ ${ }^{1}$ Department of Physics, University of Jyväskylä, P.O. Box 35 (YFL), FI-40014 Jyväskylä, Finland \\ ${ }^{2}$ Department of Physics, University of Helsinki, P.O. Box 64, FI-00014 Helsinki, Finland \\ ${ }^{3}$ Helsinki Institute of Physics, University of Helsinki, P.O. Box 64, FI-00014 Helsinki, Finland
}

(Received 10 December 2015; published 27 January 2016)

\begin{abstract}
We consider a fermionic dark matter candidate interacting via a scalar mediator coupled with the Standard Model through a Higgs portal. We consider a general setting including both scalar and pseudoscalar interactions between the scalar and fermion, and illustrate the relevant features for dark matter abundance, direct search limits and collider constraints. The case where dark matter has a self-interaction strength $\left\langle\sigma_{V}\right\rangle / m_{\psi} \sim 0.1-1 \mathrm{~cm}^{2} / \mathrm{g}$ is strongly constrained, in particular by the big bang nucleosynthesis. We show that these constraints can be alleviated by introducing a new light sterile neutrino $N$. The allowed region for the extended model consists of two triangles as a function of the mass $m_{N}$ and a sufficiently small sterile-active neutrino mixing angle $\sin \theta<0.007$ : first $10(\sin \theta)^{-2 / 5} \mathrm{MeV} \lesssim m_{N} \lesssim 1 \mathrm{GeV}$ and second $15(\sin \theta)^{-2 / 5} \mathrm{keV} \lesssim m_{N} \lesssim 1 \mathrm{MeV}$.
\end{abstract}

DOI: $10.1103 /$ PhysRevD.93.015016

\section{INTRODUCTION}

Recently there has been a lot of interest in models with extended matter sectors very weakly coupled with the Standard Model (SM) fields, see e.g. [1-11]. These are mainly motivated by the need to explain the dominating dark matter (DM) component of the energy density of the Universe and simultaneously hiding the beyond SM fields from the direct searches at collider experiments. Alternatively, the extra matter fields coupled with the SM scalar sector also affect the properties of the electroweak phase transition and enhance the possibility of successful electroweak baryogenesis.

The simplest possibility of a hidden sector consists of a single singlet scalar coupled with the SM via the Higgs portal, see e.g. [1,12-14] for DM studies and [15,16] for aspects of electroweak phase transition. While the singlet scalar can in principle act as DM and lead to a strong first order phase transition, in light of present constraints for the DM abundance and from the direct search experiments, these features cannot be realized simultaneously [14]. To alleviate this issue one is led to consider more complex hidden sector structures. The possibilities of singlet fermions and vectors have been considered in the literature [5-11]. For example in the case of singlet fermion DM interacting with the SM via the Higgs portal one can easily provide sufficient DM abundance and simultaneously use the scalar mediator to provide for strongly first order electroweak phase transition [8].

Further motivations for extended hidden sectors arise from the possibility of DM self-interactions as has been

\footnotetext{
*kimmo.kainulainen@jyu.fi

kimmo.i.tuominen@helsinki.fi

‡ville.vaskonen@jyu.fi
}

studied in various contexts [17-37]. Such interactions would allow one to reconcile the tensions between the observations and simulations of the small-scale structure of collisionless cold DM (CCDM): First, as the observations of dwarf galaxies confirm, the DM density profile is not cusped towards the center of the galaxy as seen in simulations of CCDM [38] but exhibits a flat core [39,40]. Second, the socalled missing satellite problem [41-43] and the too big to fail problem [44] can also be explained by sizable interactions between the DM particles, although alternative astrophysical explanations also exist [45-51].

In this paper we consider a simple paradigm for selfinteracting DM: a singlet fermion $\psi$ interacting via scalar mediator $s$. We consider in particular the parameter region where the scalar mediator $s$ is light. In [22] it has been pointed out that self-interactions of a DM particle $\psi$ mediated with a light force carrier $s$ and satisfying $\left(m_{\psi} / 10 \mathrm{GeV}\right)\left(m_{s} / 100 \mathrm{MeV}\right)^{2} \sim 1$, can provide the flat density profile around the core of dwarf galaxies for a wide range of interaction strengths between $\psi$ and $s$ while evading constraints on self-interactions from galactic and cluster scales. However, there exist additional stringent constraints. Most notably, if the scalar $s$ couples to the SM via the Higgs portal [2,52-58], it must decay before the onset of the big bang nucleosynthesis (BBN) around $t \sim 1 \mathrm{sec}$ in order that the success of the BBN explaining the abundances of light elements is not endangered. This sets a lower bound on the strength of coupling between the hidden and visible sectors which is too strong [31] in light of present constraints for the invisible Higgs decay at LHC and direct search constraints from the LUX experiment. Generic ways to evade this problem were proposed in [59].

Our paper can be viewed as a significant complement to earlier literature on singlet fermion DM [5-8,59]: We 
investigate the effects of both scalar and pseudoscalar couplings between the DM fermion and the scalar mediator. Via Monte Carlo scan, we probe wide mass scales for the singlet sector especially focusing on the range where the DM self-interaction cross section is large enough to reconcile the observations and simulations of small scale structure. In addition, we take systematically into account the phenomenological constraints from DM abundance and direct detection as well as theoretical constraints of stability and perturbativity. Furthermore, while we confirm that the BBN constraint can be resolved in this model via the mechanism proposed in [59], we demonstrate that recombination provides an ultimate bound on this type of scenario and the parameter space where the generic solution proposed in [59] works is very narrow.

The paper is organized as follows: In Sec. II we define the model and outline the basic computations constraints on DM abundance and direct detection. In Sec. III we study the strength of DM self-interactions and its relation to the mass of the scalar mediator. The consequences of the existence of a light mediator on the physics of the early Universe is discussed in Sec. IV. In Sec. V we present our conclusions and outlook.

\section{MODEL}

\section{A. Scalar sector and the dark matter Lagrangian}

We consider an extended scalar sector described by the potential

$$
\begin{aligned}
V(\phi, s)= & \mu_{\phi}^{2} \phi^{\dagger} \phi+\lambda_{\phi}\left(\phi^{\dagger} \phi\right)^{2}+\mu_{1} s+\frac{\mu_{s}^{2}}{2} s^{2}+\frac{\mu_{3}}{3} s^{3}+\frac{\lambda_{s}}{4} s^{4} \\
& +\mu_{\phi s}\left(\phi^{\dagger} \phi\right) s+\frac{\lambda_{\phi s}}{2}\left(\phi^{\dagger} \phi\right) s^{2},
\end{aligned}
$$

which provides typical scalar portals between the hidden singlet sector and the SM Higgs. The field $\phi$ is the usual SM Higgs doublet and $s$ is a real singlet scalar. The Higgs doublet is written in terms of its components as

$$
\phi=\left(\begin{array}{c}
\chi^{+} \\
\frac{1}{\sqrt{2}}(v+h+\mathrm{i} \chi)
\end{array}\right)
$$

where $v$ is the vacuum expectation value which at $T=0$ has the value $v=246 \mathrm{GeV}$. The stability of the tree level potential requires that

$$
\lambda_{\phi}>0, \quad \lambda_{s}>0, \quad \lambda_{\phi s}>-2 \sqrt{\lambda_{\phi} \lambda_{s}} .
$$

To further constrain the parameters we also require that the electroweak broken minimum is the deepest one at $T=0$. There are several inequivalent minima whose relative ordering can evolve as a function of temperature. As shown in [8] this potential leads to strong first order electroweak transition over a large portion of the parameter space already at the simple mean field level.

In this paper we do not consider the electroweak transition in detail, but focus on the properties of the DM candidate, which we take to be a singlet fermion described by the Lagrangian

$$
\mathcal{L}_{\mathrm{DM}}=\bar{\psi}\left(\mathrm{i} \not \partial-m_{\psi}\right) \psi+s \bar{\psi}\left(g_{s}+\mathrm{i} g_{p} \gamma_{5}\right) \psi .
$$

Without loss of generality, we can shift $s \rightarrow s^{\prime}=s-\langle s\rangle$ such that at the electroweak broken minimum $\left\langle s^{\prime}\right\rangle=0$. Since we are not assuming any discrete symmetries for the singlet scalar, the shift will simply reproduce all terms already present in the scalar potential with redefined coefficients. Hence practically we use (2.1) and (2.4), where the mass of the DM fermion at $T<T_{\mathrm{EW}}$ is given directly by the mass parameter $m_{\psi}$ and the mixing between $\phi$ and $s$ fields is determined solely by $\mu_{\phi s}$. In principle $m_{\psi}$ is a complex number, but we can always make it real by a chiral rotation on $\psi$ in Eq. (2.4). ${ }^{1}$

We use the extremization conditions to set

$$
\mu_{\phi}^{2}=-v^{2} \lambda_{\phi}, \quad \mu_{1}=-\frac{v^{2} \mu_{\phi s}}{2} .
$$

Diagonalizing the mass matrix

$$
M^{2}=\left(\begin{array}{cc}
2 v^{2} \lambda_{\phi} & v \mu_{\phi s} \\
v \mu_{\phi s} & \frac{v^{2} \lambda_{\phi s}}{2}+\mu_{s}^{2}
\end{array}\right),
$$

we trade the parameters $\lambda_{\phi}$ and $\mu_{s}^{2}$ with physical masses $m_{H}=126 \mathrm{GeV}$ and $m_{S}$,

$\lambda_{\phi}=\frac{1}{4 v^{2}}\left(m_{H}^{2}+m_{S}^{2} \pm \sqrt{\left(m_{H}^{2}-m_{S}^{2}\right)^{2}-4 \mu_{\phi s}^{2} v^{2}}\right)$,

$\mu_{S}^{2}=\frac{1}{2}\left(m_{H}^{2}+m_{S}^{2}-\lambda_{\phi s} v^{2} \mp \sqrt{\left(m_{H}^{2}-m_{S}^{2}\right)^{2}-4 \mu_{\phi s}^{2} v^{2}}\right)$.

The scalar mass eigenstates at $T=0$ are

$H=h \cos \beta+s \sin \beta, \quad S=-h \sin \beta+s \cos \beta$,

where $\tan 2 \beta=-4 v \mu_{\phi s} /\left(2 \mu_{s}^{2}+\lambda_{\phi s} v^{2}-4 \lambda_{\phi} v^{2}\right)$. Hence, the free parameters are $m_{\psi}, m_{S}, \mu_{3}, \mu_{\phi s}, \lambda_{s}, \lambda_{\phi s}, g_{s}$ and $g_{p}$. From Eq. (2.7) it follows that $\left|\mu_{\phi s}\right|<\max \left(m_{H}\right.$, $\left.m_{S}\right)^{2} / 2 v$.

\footnotetext{
${ }^{1}$ Alternatively, we could rotate away either of the couplings $g_{s, p}$, but then we would need to allow for complex $m_{\psi}$.
} 


\section{B. Constraints}

The Higgs coupling data from the LHC and Tevatron experiments constrains the mixing angle $\cos \beta$ and the decay width of the Higgs boson. The invisible decay width of the Higgs is given by

$$
\begin{aligned}
\Gamma_{\text {inv }}= & \frac{\lambda_{H S S}^{2} v_{S}}{32 \pi m_{H}} \Theta\left(m_{H}-2 m_{S}\right) \\
& +\frac{m_{H} \sin ^{2} \beta}{4 \pi}\left(g_{s}^{2} v_{\psi}^{3}+g_{p}^{2} v_{\psi}\right) \Theta\left(m_{H}-2 m_{\psi}\right),
\end{aligned}
$$

where $v_{a}^{2} \equiv 1-4 m_{a}^{2} / m_{H}^{2}$ and the coupling $\lambda_{H S S}$ is given in the Appendix. We perform a $\chi^{2}$ fit to the Higgs boson signal strength data from the LHC and Tevatron experiments [60-66] and require that the model remains within $2 \sigma$ of the best fit. Moreover, we check that the model is compatible with the electroweak precision measurements using the $S$ and $T$ parameters $[67,68]$.

We also study the perturbativity of the model as a function of the energy scale. To be concrete, we require that all models remain perturbative up to $10 \mathrm{TeV}$, but we find that many models remain perturbative up to Planck scale. The one-loop $\beta$ functions for the Yukawa couplings, $g_{s}$ and $g_{p}$, are

$$
\begin{aligned}
16 \pi^{2} \beta_{g_{s}} & =5 g_{s}^{3}+3 g_{p}^{2} g_{s}, \\
16 \pi^{2} \beta_{g_{p}} & =3 g_{p}^{3}+5 g_{s}^{2} g_{p},
\end{aligned}
$$

whereas the $\beta$ functions of the quartic couplings read

$$
\begin{aligned}
16 \pi^{2} \beta_{\lambda_{\phi}}= & 24 \lambda_{\phi}^{2}+\frac{1}{2} \lambda_{\phi s}^{2}-3\left(3 g_{\mathrm{L}}^{2}+g_{Y}^{2}-4 y_{t}^{2}\right) \lambda_{\phi} \\
+ & \frac{3}{8}\left(3 g_{\mathrm{L}}^{4}+2 g_{\mathrm{L}}^{2} g_{Y}^{2}+g_{Y}^{4}\right)-6 y_{t}^{4} \\
16 \pi^{2} \beta_{\lambda_{\phi s}}= & 4 \lambda_{\phi s}^{2}+\left(12 \lambda_{\phi}+6 \lambda_{s}\right) \lambda_{\phi s} \\
& -3\left(\frac{3}{2} g_{\mathrm{L}}^{2}+\frac{1}{2} g_{Y}^{2}-2 y_{t}^{2}-\frac{4}{3} g_{s}^{2}\right) \lambda_{\phi s} \\
16 \pi^{2} \beta_{\lambda_{s}}= & 18 \lambda_{s}^{2}+2 \lambda_{\phi s}^{2}+8 \lambda_{s} g_{s}^{2}-6\left(g_{s}^{2}+g_{p}^{2}\right)^{2}
\end{aligned}
$$

The $\beta$ functions for the top-coupling $y_{t}$ and the weak and hypercharge gauge couplings $g_{L}$ and $g_{Y}$ are standard, see for example Ref. [69].

Although one can study if the model remains perturbative and maintains vacuum stability all the way to the Planck scale, the question is not obviously meaningful as the approximation of neglecting curvature effects may not be valid [70]. Hence, we find it more natural to treat this dark sector as a low energy realization of some more complete theory. The relevant matching scale is of course completely unknown at this stage; a possible concrete UV completion is for example the model introduced in [71].
Finally, direct DM searches give an upper limit on DM scattering off nuclei. Since $\psi$ interacts with SM only through the Higgs exchange, the relevant detection channel is the spin-independent (SI) one. The nominal SI cross section in this model is given by

$$
\sigma_{\mathrm{SI}}^{0}=\frac{\mu_{N}^{2} f_{N}^{2} m_{N}^{2}}{\pi v^{2}} g_{S}^{2} \sin ^{2} \beta \cos ^{2} \beta\left(\frac{1}{m_{H}^{2}}-\frac{1}{m_{S}^{2}}\right)^{2},
$$

where $m_{N}$ is the nucleon mass, $\mu_{N}$ is the reduced mass of the WIMP-nucleon system and the Higgs-nucleon coupling $f_{N} \approx 0.303$. However, we do not require that $\psi$ forms all of the existing DM. We therefore define the relative DM relic density, $f_{\text {rel }}=\Omega h^{2} / 0.12$. Consequently, we define an effective cross section $\sigma_{\mathrm{SI}}^{\text {eff }} \equiv f_{\text {rel }} \sigma_{\mathrm{SI}}^{0}$, which is the quantity directly constrained by observations for a subleading DM component [13]. Currently the most restrictive upper limits come from the LUX and SuperCDMS experiments [72,73].

\section{Freeze-out}

To evaluate the relative DM abundance $f_{\text {rel }}$, we concentrate to the region of parameters where $\psi$ is a thermal relic that decouples with the freeze-out mechanism. ${ }^{2}$ The annihilation processes are $\psi \bar{\psi} \rightarrow H H, S S, H S$ and $\psi \bar{\psi} \rightarrow X Y$, where $X, Y$ are SM fermions or gauge bosons. The latter are $s$ channel processes with $H$ or $S$ propagators. We calculate the cross section in terms of the off-shell Higgs boson decay width:

$$
\begin{aligned}
v_{\psi s} \sigma_{\psi \bar{\psi} \rightarrow X Y}= & \frac{1}{2} \sin ^{2} \beta \cos ^{2} \beta\left(g_{s}^{2} v_{\psi s}^{2}+g_{p}^{2}\right) \\
& \left|D_{H}-D_{S}\right|^{2} \sqrt{s} \Gamma_{h}(\sqrt{s}),
\end{aligned}
$$

where $v_{\psi s}^{2} \equiv 1-4 m_{\psi}^{2} / s$ and $D_{a}^{-1} \equiv s-m_{a}^{2}+\mathrm{i} m_{a} \Gamma_{a}$. We evaluate $\Gamma_{h}(\sqrt{s})$ following [14]: for $\sqrt{s}<80 \mathrm{GeV}$ and $\sqrt{s}>300 \mathrm{GeV}$ we use perturbative decay widths taken from [74] including the QCD corrections for the quark final states. In the range $\sqrt{s} \in(80 \mathrm{GeV}, 300 \mathrm{GeV})$ we use the tabulated results from [75], which include also four body final states. As shown in [76] there are large uncertainties in the range $\sqrt{s} \in\left(2 m_{\pi}, 2 m_{c}\right)$. In this region we use the perturbative spectator approach [74]. The cross section for the scalar final states is given in the Appendix.

Note that the annihilation cross section contains contributions both from the scalar and pseudoscalar couplings $g_{s}$ and $g_{p}$, while the cross section for DM scattering on a nucleus is proportional only to $g_{s}$. This allows one to escape the direct detection limits by reducing $g_{s}$ while maintaining the large enough annihilation cross section by adjusting the value of $g_{p}$.

\footnotetext{
${ }^{2}$ Another possibility, requiring much smaller couplings $g_{p, s}$ than the ones considered here is that $\psi$ was ever only partially thermalized by the freeze-in mechanism.
} 
Given the cross sections, we solve the thermal relic abundance, $Y \equiv n_{\psi} / s$ (where $s$ is the entropy density) from the Zeldovish-Okun-Pikelner-LeeWeinberg equation $[77,78]$ :

$$
\frac{\mathrm{d} Y}{\mathrm{~d} x}=Z\left(Y^{2}-Y_{\mathrm{eq}}^{2}\right) .
$$

Here $x \equiv m_{\psi} / T$ and

$$
Z(x)=-\sqrt{\frac{\pi}{45} g_{*}(T)} \frac{M_{\mathrm{Pl}} m_{\psi}}{x^{2}}\langle v \sigma\rangle,
$$

where

$$
g_{*}^{1 / 2}(T)=\frac{h_{\text {eff }}}{\sqrt{g_{\text {eff }}}}\left(1+\frac{T}{3 h_{\text {eff }}} \frac{\mathrm{d} h_{\text {eff }}}{\mathrm{d} T}\right)
$$

and $g_{\text {eff }}$ and $h_{\text {eff }}$ are the energy and entropy degrees of freedom, respectively. For the averaged cross sections, we use the integral expression [79]

$$
\langle v \sigma\rangle=\frac{1}{8 m_{\psi}^{4} T K_{2}^{2}(x)} \int_{4 m_{\psi}^{2}}^{\infty} \mathrm{d} s \sqrt{s}\left(s-4 m_{\psi}^{2}\right) K_{1}\left(\frac{\sqrt{s}}{T}\right) \sigma_{\text {tot }}(s),
$$

where $K_{i}(y)$ are the modified Bessel functions of the second kind. Finally the relative DM relic density, $f_{\text {rel }}=\Omega h^{2} / 0.12$, is given by

$$
f_{\text {rel }}=2.30 \times 10^{9} \frac{m_{\psi}}{\mathrm{GeV}} Y\left(x_{0}\right),
$$

where $x_{0}=m_{\psi} / T_{0}$ and $T_{0}$ is the photon temperature today.

\section{DARK MATTER SELF-INTERACTION}

The DM self-interaction processes are $\psi \bar{\psi} \rightarrow \psi \bar{\psi}, \overline{\psi \psi} \rightarrow$ $\overline{\psi \psi}$ and $\psi \psi \rightarrow \psi \psi \psi$. The first of these arises from the $s$ and the $t$ channel, and the latter two from $t$ and $u$ channel diagrams with $H$ and $S$ propagators. It turns out that to obtain reasonably strong DM self-interaction $S$ has to be very light, $m_{S} \lesssim 1 \mathrm{GeV}$, and the contribution of diagrams with $H$ propagator can be neglected. In the interesting region the Born approximation for the self-interaction fails and we must calculate the cross section more accurately by solving the Schrödinger equation for Yukawa potential,

$$
V(r)=-\frac{\alpha}{r} e^{-m_{S} r},
$$

where $\alpha=\cos ^{2} \beta g_{s}^{2} / 4 \pi$. In the partial wave analysis the scattering amplitude written in terms of phase shifts $\delta_{l}$ is given by

$$
f(\theta)=\frac{1}{k} \sum_{l=0}^{\infty}(2 l+1) e^{\mathrm{i} \delta_{l}} P_{l}(\cos \theta) \sin \delta_{l},
$$

where $k=\mu v=m_{\psi} v / 2, v$ is the relative velocity and $P_{l}$ is the $l$ th Legendre polynomial. To find the phase shifts we need to solve the radial Schrödinger equation,

$\frac{1}{r^{2}} \frac{\mathrm{d}}{\mathrm{d} r}\left(r^{2} \frac{\mathrm{d} R_{l}}{\mathrm{~d} r}\right)+\left(k^{2}-\frac{l(l+1)}{r^{2}}-2 \mu V(r)\right) R_{l}=0$,

and match the solution onto the asymptotic solution,

$$
\lim _{r \rightarrow \infty} R_{l} \propto \cos \delta_{l} j_{l}(k r)-\sin \delta_{l} n_{l}(k r),
$$

where $j_{l}$ and $n_{l}$ are the spherical Bessel and spherical Neumann functions, respectively. In the numerical solution we use the method described in [29].

Combining all self-interaction processes the total differential cross section is finally given by

$$
\frac{\mathrm{d} \sigma_{\psi}}{\mathrm{d} \Omega}=2|f(\theta)-f(\pi-\theta)|^{2}+|f(\theta)|^{2},
$$

where the first term comes from the $\psi \psi \psi$ - and $\bar{\psi} \overline{\text {-channels, }}$ which need to be antisymmetrized over the scattering angle $\theta$, and the last term represents the $\bar{\psi} \psi$-channel.

The self-interactions of DM particles are essential input for numerical simulations of DM halo formation. The scattering angle dependence of the differential cross section is particularly important when the particle trajectories are tracked through collisions. Indeed, while the integrated cross section is strongly enhanced in the forward region for light mediators, the forward collisions are unimportant as they leave the particle trajectories unaffected. Hence, to parametrize transport properties one uses instead the viscosity cross section,

$$
\sigma_{V}=\int \mathrm{d} \Omega \sin ^{2} \theta \frac{\mathrm{d} \sigma_{\psi}}{\mathrm{d} \Omega},
$$

which in general is some function of the relative velocity of the DM particles. Moreover, we average the viscosity cross section over the thermal DM velocity distribution:

$$
\left\langle\sigma_{V}\right\rangle=\int \frac{\mathrm{d}^{3} v}{\left(4 \pi \Delta v^{2}\right)^{\frac{3}{2}}} e^{-v^{2} / 4 \Delta v^{2}} \sigma_{V},
$$

where $\Delta v$ is the radial velocity dispersion of the halo. We use $\Delta v=10 \mathrm{~km} / \mathrm{sec}$, corresponding to observations of stellar motions in local dwarf galaxies [80]. In Fig. 1 we show the dependence of the self-interaction cross section on $m_{S}$ for fixed parameters. In order to be consistent with astrophysical observations, cosmological simulations require $\left\langle\sigma_{V}\right\rangle / m_{\psi} \sim 0.1-1 \mathrm{~cm}^{2} / \mathrm{g}$ [24,25,27], and this region is shown as a shaded band in Fig. 1. 


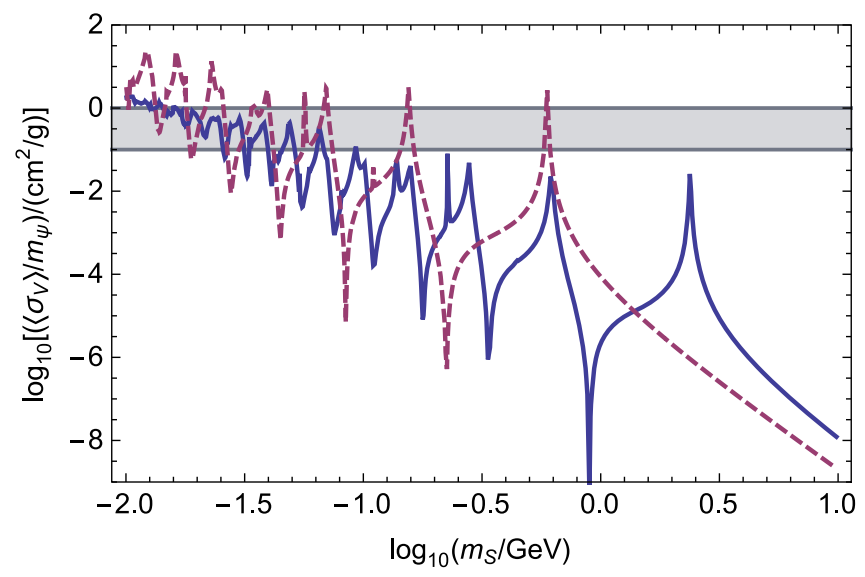

FIG. 1. Self-interaction as a function of $m_{S}$ for fixed $\alpha=0.01$ and $\Delta v=10 \mathrm{~km} / \mathrm{sec}$. Solid and dashed lines correspond to $m_{\psi}=400 \mathrm{GeV}$ and $m_{\psi}=100 \mathrm{GeV}$, respectively. The shaded band corresponds to $\left\langle\sigma_{V}\right\rangle / m_{\psi} \sim 0.1-1 \mathrm{~cm}^{2} / \mathrm{g}$.

If $m_{S}<m_{H} / 2$ the LHC limits on the invisible Higgs decays constrain the parameter space significantly. Also the spin-independent DM-nucleon cross section, $\sigma_{\mathrm{SI}}$, which is proportional to $1 / m_{S}^{4}$, gives a strong constraint. For a very light $S$ the latter is by far the most stringent of the two. Avoiding these limits requires a very small effective coupling $g_{s} \sin \beta$ when $S$ is very light. However, $g_{s}$ itself should not be small, because we want to have a large selfinteraction rate. It follows that the mixing angle $\beta$ has to be very small.

In order to study the feasibility of the model, we performed a Monte Carlo scan of the parameter space with the following priors:

$$
\begin{gathered}
\left|g_{s}\right|<1, \quad\left|g_{p}\right|<1, \quad 0<\lambda_{s}<\pi, \\
-9<\log \left|\lambda_{\phi s}\right|<0,
\end{gathered}
$$

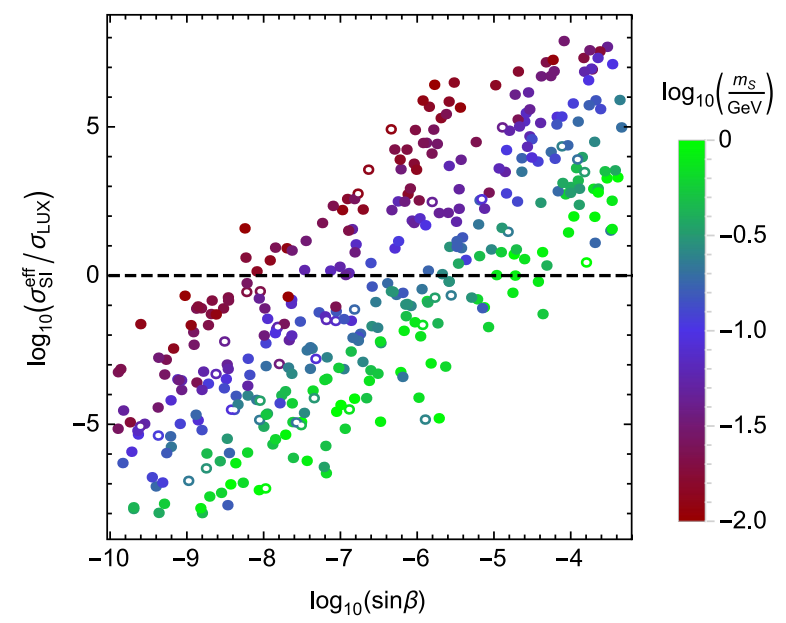

and

$$
\begin{aligned}
-9 & <\log \left|\mu_{\phi s} / \mathrm{GeV}\right|<1.5, \\
-5 & <\log \left|\mu_{3} / \mathrm{GeV}\right|<0 \\
1 \mathrm{GeV} & <m_{\psi}<1000 \mathrm{GeV}, \\
-2 & <\log \left(m_{S} / \mathrm{GeV}\right)<0 .
\end{aligned}
$$

The resulting parameter sets, which are in agreement with the collider constraints, are shown in Fig. 2 as a function of the mixing angle $\sin \beta$ and the SI-detection cross section normalized to LUX sensitivity (left) and as a function of the DM particle mass $m_{\psi}$ and self-interaction cross section (right). The color coding of dots specifies the mediator mass $m_{S}$ as indicated by the vertical bar to the right of the panels. To be consistent with the LUX limit [72] the mixing angle has to roughly satisfy a constraint

$$
\sin \beta \lesssim 10^{-5}\left(m_{S} / \mathrm{GeV}\right)^{2},
$$

consistent with Eq. (2.15).

\section{COSMOLOGY OF A LIGHT LONG-LIVED MEDIATOR}

We found that in the region interesting for DM selfinteractions the mediator is light, $m_{S} \lesssim 0.1 \mathrm{GeV}$, and its coupling to SM fermions is very small, $\sin \beta \lesssim 10^{-5}$. Since $S$ decay to SM particles is suppressed by the small mixing angle, its lifetime tends to be very long and it may cause problems in the early Universe [31,81]. In particular there will be an upper bound on the $S$-lifetime from nucleosynthesis.

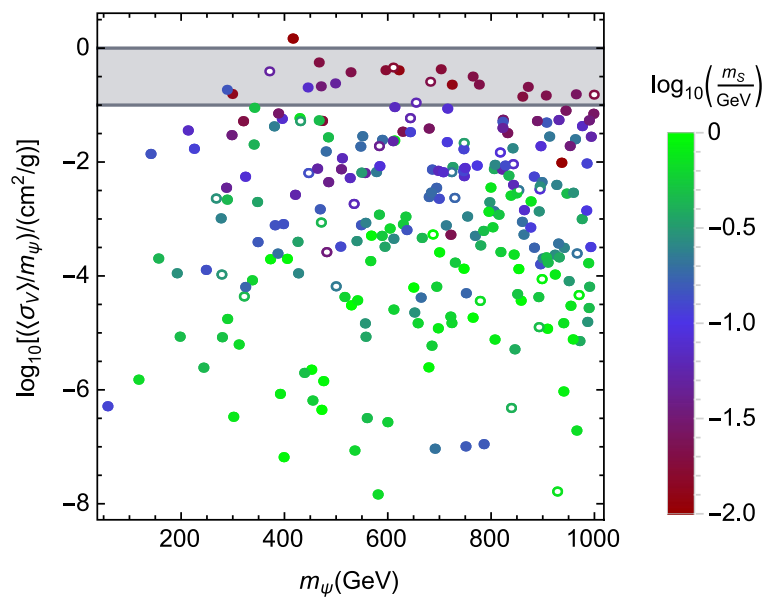

FIG. 2. Shown are the parameter sets which are in agreement with collider constraints and give a relic density in the range $0.8<f_{\text {rel }} \leq 1$. In the right panel all points are below the LUX limit. Unfilled points indicate models that remain perturbative up to Planck scale. 


\section{A. Bound from the big bang nucleosynthesis}

We will first discuss the BBN bound in the case where $s$ decays only to SM particles. Here $S$ decays predominantly to the heaviest available SM fermion with a width:

$$
\Gamma_{S \rightarrow f \bar{f}}=\frac{m_{f}^{2} \sin ^{2} \beta}{8 \pi v^{2}} m_{S}\left(1-\frac{4 m_{f}^{2}}{m_{S}^{2}}\right)^{3 / 2},
$$

where $v \approx 246 \mathrm{GeV}$ and $m_{f}$ is the mass of the fermion. This corresponds to a lifetime

$$
\tau_{S} \approx\left(\frac{10^{-6}}{\sin \beta}\right)^{2}\left(\frac{\mathrm{MeV}}{m_{f}}\right)^{2}\left(\frac{\mathrm{GeV}}{m_{S}}\right) \mathrm{sec} .
$$

For $m_{S} \gtrsim 2 m_{\mu}, S$ decays to muons and for $2 m_{\mu}>m_{S}>$ $2 m_{e}$ the dominant channel is to electrons and positrons.

When $S$ decays to SM particles, the decay products in general thermalize quickly. An exception may be neutrinos, which decouple from equilibrium at $T \approx 4.3 \mathrm{MeV}$ (muonand tau neutrinos) and $T \approx 2.6 \mathrm{MeV}$ (electron neutrinos) [82]. Thus, if decays take place around $2.3 \mathrm{MeV}$, corresponding to $\tau_{S} \gtrsim 0.1 \mathrm{sec}$, they may lead to an increase of the electron neutrino density [83]. This would increase the rate of reactions $\nu_{e}+n \leftrightarrow p+e^{-}$and $\bar{\nu}_{e}+p \leftrightarrow n+e^{+}$, which keep protons and neutrons in thermal equilibrium and hence decrease the ${ }^{4} \mathrm{He}$ abundance [84]. For longer lifetimes this neutrino heating effect becomes rapidly weaker. Instead, for $\tau_{S} \gtrsim 1 \mathrm{sec}$, the residual population of $S$-particles starts to increase the expansion rate of the Universe. This causes the proton-neutron equilibrium reactions to drop out of equilibrium earlier, which makes the ${ }^{4} \mathrm{He}$ abundance larger. A fully quantitative analysis of these effects is beyond the scope of this paper. Instead we follow [85] and, to remain within the $2 \sigma$ limit of the observed ${ }^{4} \mathrm{He}$ abundance, require that $\tau_{S}<1 \mathrm{sec}$.

Imposing the limit (3.10) on the mixing angle, Eq. (4.2) gives a rough bound on the $S$-particle lifetime:

$$
\tau_{S} \gtrsim 0.01\left(\frac{\mathrm{MeV}}{m_{f}}\right)^{2}\left(\frac{\mathrm{GeV}}{m_{S}}\right)^{5} \mathrm{sec} .
$$

Since for $m_{S} \lesssim 0.2 \mathrm{GeV}$ the dominant decay channel is to electrons, it is clear that a light mediator with $m_{S} \lesssim 0.1 \mathrm{GeV}$, decaying to SM particles, is not consistent with the BBN and direct search constraints. This conclusion becomes evident also from Fig. 3, where the bounds on the mixing angle and the mediator mass following from the BBN and LUX constraints are shown more accurately.

\section{B. Extension with a light sterile neutrino}

An obvious solution to alleviate this problem is to introduce new states into which a light $S$ can decay to, such as a light sterile neutrino $N$ which couples to the singlet scalar via a Lagrangian [59],

$$
\mathcal{L}_{\mathrm{SNN}}=y_{N} S N \bar{N} .
$$

If $m_{N}<m_{S} / 2$ and $N$ mixes with the SM neutrinos with a small, but finite mixing angle $\sin \theta$, it is possible to extend the acceptable parameter space. Let us now consider in quantitative detail how this mechanism works in this model.

First, an upper bound for the mixing $\sin \theta$ is obtained from the invisible decay width of the $Z$ boson, experimentally determined to be [68]
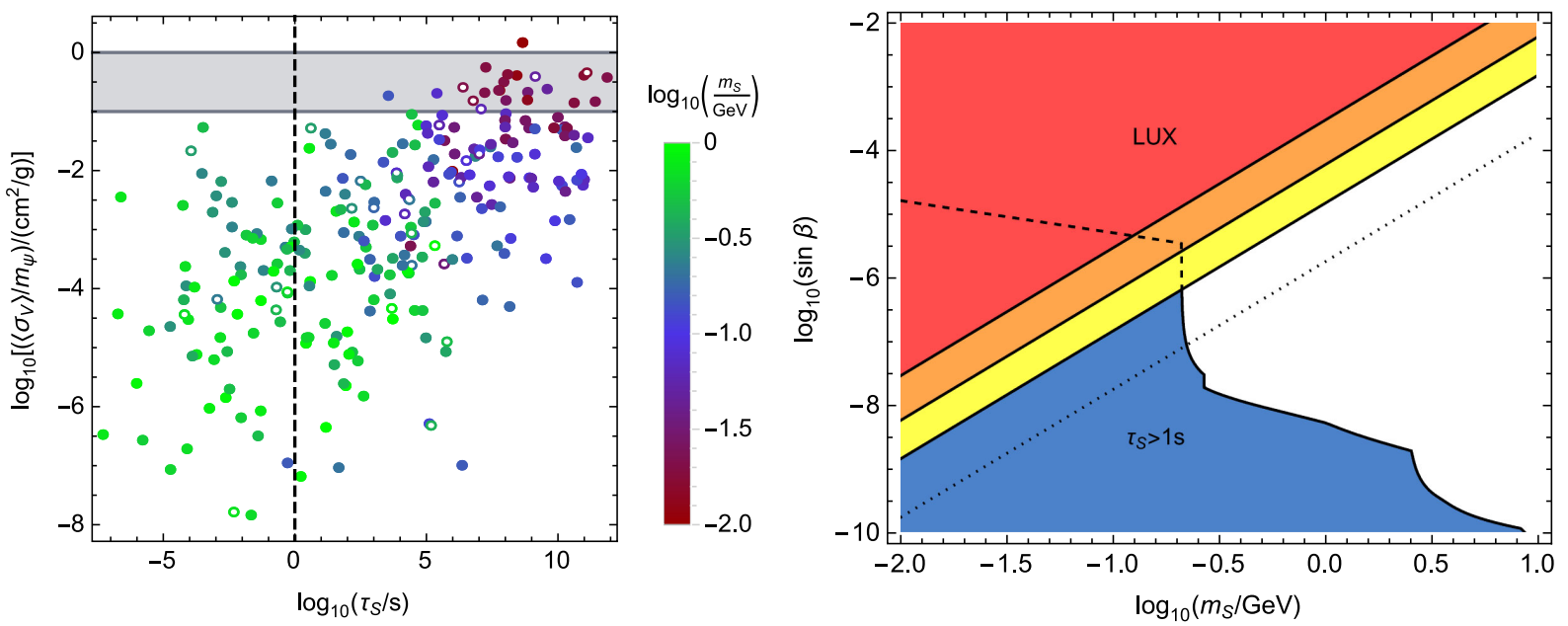

FIG. 3. Left: Shown are the models which are in agreement with collider constraints and the LUX limit and give $0.8<f_{\text {rel }} \leq 1$ as a function of the $S$-lifetime and the self-interaction cross section $\left\langle\sigma_{V}\right\rangle / m_{\psi}$. The vertical dotted line shows the $\tau_{S}=1$ sec limit and the shaded region corresponds to models with sufficient self-interactions. Unfilled points again indicate models that remain perturbative up to Planck scale. Right: The excluded regions in the mixing angle versus the scalar mass from BBN (blue area) and from the LUX constraints (yellow, orange, red) corresponding to $g_{s}=0.4,0.1,0.02$, respectively. The DM mass is fixed to $400 \mathrm{GeV}$. The dotted line indicates the expected XENON1T experiment reach [86] in the case $g_{s}=0.4$. 


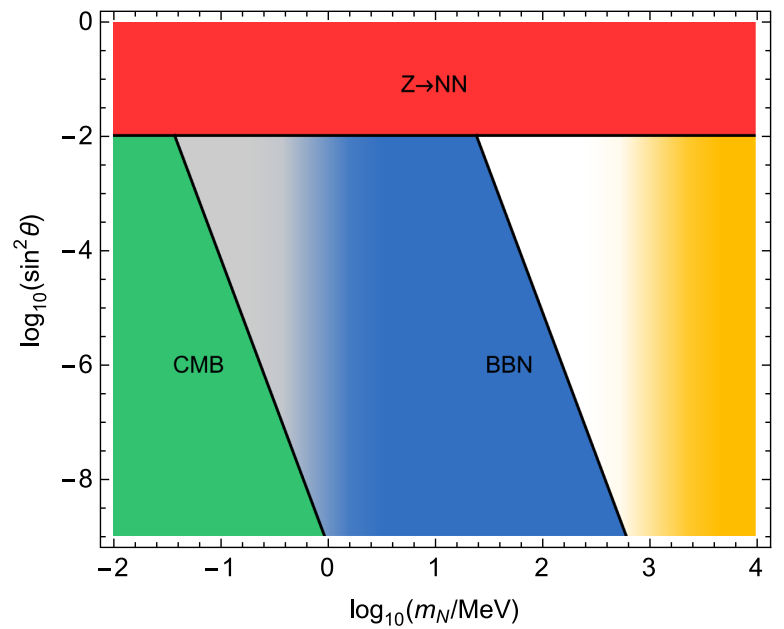

FIG. 4. Shown are the excluded regions in the sterile $N$ parameters due to the $Z$-decay width (red area), the BBN (blue area) and the $\mathrm{CMB}$ (green area). In the rightmost shaded region (yellow area) $S$ is too heavy to provide a sufficiently strong selfinteraction for $\psi$.

$$
\frac{\Gamma(Z \rightarrow \text { inv })}{\Gamma(Z \rightarrow \nu \nu)}=2.990 \pm 0.007
$$

If we require that the contribution from the sterile neutrino decay channel is less than $1 \sigma$ we get $\sin ^{2} \theta<0.007$. This limit is shown by the red area in Fig. 4.

Second, we require that the $S$ decays to sterile neutrinos sufficiently fast and also that the sterile neutrinos have time to decay to SM neutrinos before they freeze out. To be precise, we first require that the lifetimes of both $S$ and $N$ are less than $0.1 \mathrm{sec}$. The decay width for the process $S \rightarrow$ $N \bar{N}$ is the same as in the SM fermion case:

$$
\Gamma_{S \rightarrow N \bar{N}}=\frac{y_{N}^{2}}{16 \pi} m_{S}\left(1-\frac{4 m_{N}^{2}}{m_{S}^{2}}\right)^{3 / 2},
$$

so the lifetime of $S$ is smaller than $0.1 \mathrm{sec}$ if

$$
y_{N} \gtrsim 2 \times 10^{-11}\left(\frac{\mathrm{GeV}}{m_{S}}\right)^{1 / 2} .
$$

Clearly $y_{N}$ be arranged such that $S$ decays fast enough. We have checked explicitly that the new annihilation channel induced by this coupling does not affect the DM abundance. On the other hand, the main decay channel for the sterile neutrino is to three SM neutrinos. The decay width for this process is

$$
\Gamma_{N \rightarrow 3 \nu}=\frac{G_{F}^{2}}{192 \pi^{2}} m_{N}^{5} \sin ^{2} \theta .
$$

The constraint that the lifetime of $N$ is smaller than $0.1 \mathrm{sec}$ is then expressed as

$$
m_{N}(\sin \theta)^{2 / 5}>10 \mathrm{MeV} \text {. }
$$

Combining this with the $Z$-invisible width bound shows that $m_{N}>26.5 \mathrm{MeV}$. Hence adding a new sterile neutrino $N$ and requiring it to decay to SM neutrinos before they drop out of thermal equilibrium relieves the BBN limit for $m_{S}>53 \mathrm{MeV}$. Nominally, this limit excludes the region to the left from the rightmost solid line in Fig. 4.

However, if decays $S \rightarrow N \bar{N}$ occur early enough to allow for a sufficient subsequent dilutation of the $N$-population, $N$ may well be essentially stable during the nucleosynthesis. Its residual energy density would still increase the expansion rate causing an earlier decoupling of $n p$ reactions and leaving less time for free neutron decay. Both effects increase the helium abundance. In the case of relativistic neutrino the strength of the first is roughly twice as strong as that of the other. The effect of $N$ on BBN, counted in equivalent relativistic neutrino degrees of freedom, can then be estimated by

$$
\Delta N_{\mathrm{eff}}\left(m_{N}\right) \approx \frac{2}{3} \frac{\rho_{N}\left(T_{\mathrm{np}}^{N}\right)}{\rho_{\nu}\left(T_{\mathrm{np}}^{\nu}\right)}+\frac{1}{3} \frac{\rho_{N}\left(T_{\mathrm{n}}^{N}\right)}{\rho_{\nu}\left(T_{\mathrm{n}}^{\nu}\right)},
$$

where $\rho_{\nu}\left(T_{\nu}\right) \approx 3.15 T_{\nu} n_{\nu}\left(T_{\nu}\right) \quad$ and $\rho_{N}\left(T_{N}\right) \approx\left(m_{N}+\right.$ $\left.\frac{3}{2} T_{N}\right) n_{N}\left(T_{N}\right)$. Assuming that $N$-species decoupled while relativistic above QCD transition, which is the case already for a still rather modest $y_{N} \gtrsim 3 \times 10^{-9}$, the dilutation factor of $N$-species relative to neutrinos is $n_{N} / n_{\nu} \approx 43 / 303$ and consequently $T_{N} \approx 0.52 T_{\nu}$. Then, using $T_{\mathrm{np}}^{\nu} \approx 0.6 \mathrm{MeV}$ and $T_{\mathrm{n}}^{\nu} \approx(4 / 11) \times 0.07 \mathrm{MeV}$ we find that $\Delta N_{\text {eff }} \approx 0.5$ for $m_{N} \approx 0.73 \mathrm{MeV}$. Allowing for the roughness of this argument, we quote the BBN constraint as a bound $m_{N} \lesssim 1 \mathrm{MeV}$.

Even in this case $N$ still has to decay before recombination, where it would otherwise completely dominate the energy density. This sets another bound on the lifetime $\tau_{N}<3.8 \times 10^{5} \mathrm{y}$, which gives a new lower bound on the mass and the mixing angle:

$$
m_{N}(\sin \theta)^{2 / 5}>15 \mathrm{keV} .
$$

Combining this with the Z-decay bound gives the absolute lower bound $m_{N}>41 \mathrm{keV}$, corresponding to the left borderline of the small triangular allowed region shown by the gray color in Fig. 4. Note that the constraints on the mass and mixing of $N$ are independent of the dark matter couplings, since they arise from the requirement that the lifetime of $N$ is short.

\section{CONCLUSIONS}

In this paper we have considered a model for selfinteracting DM. The DM candidate was taken to be a fermion $\psi$ with self-interactions mediated by a scalar $S$. The scalar also mediates coupling between the hidden and 
visible sectors via the usual Higgs portal. We demonstrated that over a large region of parameter space the model can saturate the observed DM relic density. For the selfinteractions to be sufficiently strong, the scalar mediator has to be light which leads to the well-known problems in the early Universe if the scalar survives until the era of BBN. We showed that the pseudoscalar coupling $g_{p}$ makes it easier to get the correct DM abundance and to simultaneously escape the direct detection limits by reducing $g_{s}$, but since the DM self-interaction cross section is dominantly proportional only to $g_{s}$, this does not alleviate the problem with DM direct detection and BBN limits in getting sufficiently strong DM self-interaction. We confirmed the observation [59] that this problem can be solved by assuming the existence of a light sterile neutrino coupling with the singlet scalar and mixing with the active neutrinos. However, a stringent lower bound on the sterile neutrino mass scale as a function of the mixing angle is provided by the requirement that the sterile neutrino has to decay before recombination. The allowed region for the extended model consists of two triangles as a function the mass $m_{N}$ and a sufficiently small sterile-active neutrino mixing angle $\sin \theta<0.007$ : first at $10(\sin \theta)^{-2 / 5} \mathrm{MeV} \lesssim$ $m_{N} \lesssim 1 \mathrm{GeV}$ and second at $15(\sin \theta)^{-2 / 5} \mathrm{keV} \lesssim m_{N} \lesssim$ $1 \mathrm{MeV}$.
Having established the viability of this model in the broad cosmological context, there are several further issues which could be addressed. A detailed investigation of various astrophysical probes for the DM candidate should be carried out. Also, as we have emphasized, the scalar sector of the model can also lead to strong first order electroweak phase transition, which is a basic requirement for successful baryogenesis. We leave the more detailed investigation of these issues within this model for future work.

\section{ACKNOWLEDGMENTS}

We acknowledge the financial support from the Academy of Finland, Projects No. 278722 and No. 267842. V. V. acknowledges the financial support from the Finnish Cultural foundation and Magnus Ehrnrooth foundation.

\section{APPENDIX: CROSS SECTIONS}

Here we give the formulas for the computation of the annihilation cross section for the model considered in Sec. II. To make the equations more concise, it is useful to define the couplings, which at the electroweak broken vacuum read

$$
\begin{gathered}
\lambda_{H H H}=-6 v \lambda_{\phi} \cos ^{3} \beta-3 \mu_{\phi s} \cos ^{2} \beta \sin \beta-3 v \lambda_{\phi s} \cos \beta \sin ^{2} \beta-2 \mu_{3} \sin ^{3} \beta, \\
\lambda_{H H S}=6 v \lambda_{\phi} \cos ^{2} \beta \sin \beta-\mu_{\phi s} \cos ^{3} \beta+2 \mu_{\phi s} \cos \beta \sin ^{2} \beta-2 v \lambda_{\phi s} \cos ^{2} \beta \sin \beta+v \lambda_{\phi s} \sin ^{3} \beta-2 \mu_{3} \sin ^{2} \beta \cos \beta, \\
\lambda_{H S S}=-6 v \lambda_{\phi} \cos \beta \sin ^{2} \beta+2 \mu_{\phi s} \cos ^{2} \beta \sin \beta-v \lambda_{\phi s} \cos ^{3} \beta-\mu_{\phi s} \sin ^{3} \beta+2 v \lambda_{\phi s} \sin ^{2} \beta \cos \beta-2 \mu_{3} \sin \beta \cos ^{2} \beta, \\
\lambda_{S S S}=6 v \lambda_{\phi} \sin ^{3} \beta-3 \mu_{\phi s} \sin ^{2} \beta \cos \beta+3 v \lambda_{\phi s} \sin \beta \cos ^{2} \beta-2 \mu_{3} \cos ^{3} \beta,
\end{gathered}
$$

and

$$
g_{s, H}=g_{s} \sin \beta, \quad g_{p, H}=g_{p} \sin \beta, \quad g_{s, S}=g_{s} \cos \beta, \quad g_{p, S}=g_{p} \cos \beta .
$$

Decay widths for the processes $H \rightarrow \psi \bar{\psi}$ and $S \rightarrow \psi \bar{\psi}$ are

$$
\Gamma_{X \rightarrow \psi \bar{\psi}}=\Theta\left(m_{X}-2 m_{\psi}\right) \frac{m_{X} R_{X}^{2}}{8 \pi}\left(g_{s}^{2}\left(1-\frac{4 m_{\psi}^{2}}{m_{X}^{2}}\right)^{\frac{3}{2}}+g_{p}^{2} \sqrt{1-\frac{4 m_{\psi}^{2}}{m_{X}^{2}}}\right)
$$

where $X=H, S$ and $R_{H}=\sin \beta, R_{S}=\cos \beta$. The cross section for the process $a b \rightarrow c d$ reads

$$
\sigma(a b \rightarrow c d)=\frac{1}{16 \pi \lambda\left(s, m_{a}^{2}, m_{b}^{2}\right)} \int_{t_{-}}^{t_{+}} \mathrm{d} t|T(a b \rightarrow c d)|^{2},
$$

where $\lambda(x, y, z)=x^{2}+y^{2}+z^{2}-2 x y-2 x z-2 y z$ and

$$
t_{ \pm}=m_{a}^{2}+m_{c}^{2} \pm \frac{\sqrt{\lambda\left(s, m_{a}^{2}, m_{b}^{2}\right) \lambda\left(s, m_{c}^{2}, m_{d}^{2}\right)}}{2 s} \pm 2 \sqrt{\left(m_{a}^{2}+\frac{\lambda\left(s, m_{a}^{2}, m_{b}^{2}\right)}{4 s}\right)\left(m_{c}^{2}+\frac{\lambda\left(s, m_{c}^{2}, m_{d}^{2}\right)}{4 s}\right)}
$$


The squared amplitude, averaged over the initial states and summed over the final states, for the DM, $\psi$, annihilating to two scalars, $h_{i}, h_{j}=H, S$, or equivalently for $h_{i}$ and $h_{j}$ annihilating to $\psi \bar{\psi}$-pair is given by

$$
\left|T_{i j}\right|^{2}=\left(1-\frac{\delta_{i j}}{2}\right)\left(T_{i j}^{(s s)}+T_{i j}^{(t t)}+T_{i j}^{(u u)}+2 T_{i j}^{(s t)}+2 T_{i j}^{(s u)}+2 T_{i j}^{(t u)}\right),
$$

where

$$
\begin{aligned}
T_{i j}^{(s s)}=\left(2-\delta_{i j}\right)\left(\left(s-4 m_{\psi}^{2}\right)\left|\sum_{k=H, S} \frac{\lambda_{i j k} g_{s, k}}{s-m_{k}^{2}+\mathrm{i} m_{k} \Gamma_{k}}\right|^{2}+s\left|\sum_{k=H, S} \frac{\lambda_{i j k} g_{p, k}}{s-m_{k}^{2}+\mathrm{i} m_{k} \Gamma_{k}}\right|^{2}\right), \\
T_{i j}^{(t t)}=\left(2\left(m_{\psi}^{4}-m_{\psi}^{2}(t+u)-m_{i}^{2} m_{j}^{2}+t u\right) g_{s, i}^{2} g_{s, j}^{2}+2\left(m_{\psi}^{4}+m_{\psi}^{2}(u-3 t)-m_{i}^{2} m_{j}^{2}+t u\right)\left(g_{s, i}^{2} g_{p, j}^{2}+g_{p, i}^{2} g_{s, j}^{2}\right)\right. \\
\left.\quad-2\left(7 m_{\psi}^{4}+m_{\psi}^{2}\left(-4 m_{i}^{2}-4 m_{j}^{2}+9 t+u\right)+m_{i}^{2} m_{j}^{2}-t u\right) g_{p, i}^{2} g_{p, j}^{2}+8 m^{2} s g_{s, i} g_{s, j} g_{p, i} g_{p, j}\right)\left(t-m_{\psi}^{2}\right)^{-2}, \\
T_{i j}^{(u u)}=\left(2\left(m_{\psi}^{4}-m_{\psi}^{2}(t+u)-m_{i}^{2} m_{j}^{2}+t u\right) g_{s, i}^{2} g_{s, j}^{2}+2\left(m_{\psi}^{4}+m_{\psi}^{2}(t-3 u)-m_{i}^{2} m_{j}^{2}+t u\right)\left(g_{s, i}^{2} g_{p, j}^{2}+g_{p, i}^{2} g_{s, j}^{2}\right)\right. \\
\left.\quad-2\left(7 m_{\psi}^{4}+m_{\psi}^{2}\left(-4 m_{i}^{2}-4 m_{j}^{2}+9 u+t\right)+m_{i}^{2} m_{j}^{2}-t u\right) g_{p, i}^{2} g_{p, j}^{2}+8 m^{2} s g_{s, i} g_{s, j} g_{p, i} g_{p, j}\right)\left(u-m_{\psi}^{2}\right)^{-2}, \\
T_{i j}^{(s t)}=\sum_{k=H, S} \frac{\lambda_{i j k}}{\left|s-m_{k}^{2}+\mathrm{i} m_{k} \Gamma_{k}\right|\left(t-m_{\psi}^{2}\right)} \times\left(\left(2 m_{\psi}(t-u) g_{s, i} g_{s, j}+2 m_{\psi}\left(4 m_{\psi}^{2}-2 m_{i}^{2}-2 m_{j}^{2}+3 t+u\right) g_{p, i} g_{p, j}\right) g_{s, k}\right. \\
\left.+\left(2 m_{\psi}\left(m_{i}^{2}-m_{j}^{2}+s\right) g_{s, i} g_{p, j}+2 m_{\psi}\left(-m_{i}^{2}+m_{j}^{2}+s\right) g_{p, i} g_{s, j}\right) g_{p, k}\right), \\
T_{i j}^{(s t)=} \sum_{k=H, S} \frac{\lambda_{i j k}}{\left|s-m_{k}^{2}+\mathrm{i} m_{k} \Gamma_{k}\right|\left(u-m_{\psi}^{2}\right)} \times\left(\left(2 m_{\psi}(u-t) g_{s, i} g_{s, j}+2 m_{\psi}\left(4 m_{\psi}^{2}-2 m_{i}^{2}-2 m_{j}^{2}+3 u+t\right) g_{p, i} g_{p, j}\right) g_{s, k}\right. \\
\left.+\left(2 m_{\psi}\left(m_{i}^{2}-m_{j}^{2}+s\right) g_{s, i} g_{p, j}+2 m_{\psi}\left(-m_{i}^{2}+m_{j}^{2}+s\right) g_{p, i} g_{s, j}\right) g_{p, k}\right), \\
T_{i j}^{(t u)}=\left(2\left(m_{\psi}^{4}-m_{\psi}^{2}(t+u)-m_{i}^{2} m_{j}^{2}+t u\right) g_{s, i}^{2} g_{s, j}^{2}-2\left(m_{\psi}^{4}+m_{\psi}^{2}(t-3 u)-m_{i}^{2} m_{j}^{2}+t u\right) g_{s, i}^{2} g_{p, j}^{2}\right. \\
\quad-2\left(m_{\psi}^{4}+m_{\psi}^{2}(u-3 t)-m_{i}^{2} m_{j}^{2}+t u\right) g_{p, i}^{2} g_{s, j}^{2}-2\left(9 m_{\psi}^{4}+m_{\psi}^{2}\left(-4 m_{i}^{2}-4 m_{j}^{2}+3(t+u)\right)-m_{i}^{2} m_{j}^{2}+t u\right) g_{p, i}^{2} g_{p, j}^{2} \\
\left.+8 m^{2} s g_{s, i} g_{s, j} g_{p, i} g_{p, j}\right)\left(t-m_{\psi}^{2}\right)^{-1}\left(u-m_{\psi}^{2}\right)^{-1} .
\end{aligned}
$$

[1] J. McDonald, Gauge singlet scalars as cold dark matter, Phys. Rev. D 50, 3637 (1994).

[2] C. Burgess, M. Pospelov, and T. ter Veldhuis, The minimal model of nonbaryonic dark matter: A singlet scalar, Nucl. Phys. B619, 709 (2001).

[3] L. Lopez Honorez, E. Nezri, J. F. Oliver, and M. H. Tytgat, The inert doublet model: An archetype for dark matter, J. Cosmol. Astropart. Phys. 02 (2007) 028.

[4] J. Ruiz-Alvarez, C. de S. Pires, F. S. Queiroz, D. Restrepo, and P. Rodrigues da Silva, On the connection of gammarays, dark matter and Higgs searches at LHC, Phys. Rev. D 86, 075011 (2012).

[5] L. Lopez-Honorez, T. Schwetz, and J. Zupan, Higgs portal, fermionic dark matter, and a Standard Model like Higgs at 125 GeV, Phys. Lett. B 716, 179 (2012).
[6] M. Fairbairn and R. Hogan, Singlet fermionic dark matter and the electroweak phase transition, J. High Energy Phys. 09 (2013) 022.

[7] A. Alves, S. Profumo, and F. S. Queiroz, The dark $Z^{\prime}$ portal: Direct, indirect and collider searches, J. High Energy Phys. 04 (2014) 063.

[8] T. Alanne, K. Tuominen, and V. Vaskonen, Strong phase transition, dark matter and vacuum stability from simple hidden sectors, Nucl. Phys. B889, 692 (2014).

[9] T. Hambye, Hidden vector dark matter, J. High Energy Phys. 01 (2009) 028.

[10] H. Davoudiasl and I. M. Lewis, Dark matter from hidden forces, Phys. Rev. D 89, 055026 (2014).

[11] S. Di Chiara and K. Tuominen, A minimal model for $\mathrm{SU}(N)$ vector dark matter, J. High Energy Phys. 11 (2015) 188. 
[12] J. McDonald, Thermally Generated Gauge Singlet Scalars as Self-interacting Dark Matter, Phys. Rev. Lett. 88, 091304 (2002).

[13] J. M. Cline and K. Kainulainen, Electroweak baryogenesis and dark matter from a singlet Higgs, J. Cosmol. Astropart. Phys. 01 (2013) 012.

[14] J. M. Cline, K. Kainulainen, P. Scott, and C. Weniger, Update on scalar singlet dark matter, Phys. Rev. D 88, 055025 (2013).

[15] S. Profumo, M. J. Ramsey-Musolf, and G. Shaughnessy, Singlet Higgs phenomenology and the electroweak phase transition, J. High Energy Phys. 08 (2007) 010.

[16] J. R. Espinosa, T. Konstandin, and F. Riva, Strong electroweak phase transitions in the standard model with a singlet, Nucl. Phys. B854, 592 (2012).

[17] D. N. Spergel and P. J. Steinhardt, Observational Evidence for Self-interacting Cold Dark Matter, Phys. Rev. Lett. 84, 3760 (2000).

[18] B. D. Wandelt, R. Dave, G. R. Farrar, P. C. McGuire, D. N. Spergel et al., Self-interacting dark matter, arXiv:astro-ph/ 0006344.

[19] A. E. Faraggi and M. Pospelov, Self-interacting dark matter from the hidden heterotic string sector, Astropart. Phys. 16, 451 (2002).

[20] R. Mohapatra, S. Nussinov, and V. Teplitz, Mirror matter as self-interacting dark matter, Phys. Rev. D 66, 063002 (2002).

[21] A. Kusenko and P. J. Steinhardt, Q ball Candidates for Selfinteracting Dark Matter, Phys. Rev. Lett. 87, 141301 (2001).

[22] A. Loeb and N. Weiner, Cores in Dwarf Galaxies from Dark Matter with a Yukawa Potential, Phys. Rev. Lett. 106, 171302 (2011).

[23] C. Kouvaris, Limits on Self-Interacting Dark Matter, Phys. Rev. Lett. 108, 191301 (2012).

[24] M. Rocha, A. H. G. Peter, J. S. Bullock, M. Kaplinghat, S. Garrison-Kimmel, J. Onorbe, and L. A. Moustakas, Cosmological simulations with self-Interacting dark matter I: Constant density cores and substructure, Mon. Not. R. Astron. Soc. 430, 81 (2013).

[25] A. H. G. Peter, M. Rocha, J. S. Bullock, and M. Kaplinghat, Cosmological simulations with self-interacting dark matter II: Halo shapes vs observations, Mon. Not. R. Astron. Soc. 430, 105 (2013).

[26] M. Vogelsberger and J. Zavala, Direct detection of selfinteracting dark matter, Mon. Not. R. Astron. Soc. 430, 1722 (2013).

[27] J. Zavala, M. Vogelsberger, and M. G. Walker, Constraining self-interacting dark matter with the Milky Way's dwarf spheroidals, Mon. Not. R. Astron. Soc. Lett. 431, L20 (2013).

[28] F. Kahlhoefer, K. Schmidt-Hoberg, M. T. Frandsen, and S. Sarkar, Colliding clusters and dark matter self-interactions, Mon. Not. R. Astron. Soc. 437, 2865 (2014).

[29] S. Tulin, H.-B. Yu, and K. M. Zurek, Beyond collisionless dark matter: Particle physics dynamics for dark matter halo structure, Phys. Rev. D 87, 115007 (2013).

[30] M. Kaplinghat, R. E. Keeley, T. Linden, and H.-B. Yu, Tying Dark Matter to Baryons with Self-interactions, Phys. Rev. Lett. 113, 021302 (2014).
[31] M. Kaplinghat, S. Tulin, and H.-B. Yu, Direct detection portals for self-interacting dark matter, Phys. Rev. D 89, 035009 (2014).

[32] J. M. Cline, Z. Liu, G. Moore, and W. Xue, Scattering properties of dark atoms and molecules, Phys. Rev. D 89, 043514 (2014).

[33] J. M. Cline, Z. Liu, G. Moore, and W. Xue, Composite strongly interacting dark matter, Phys. Rev. D 90, 015023 (2014).

[34] K. Petraki, L. Pearce, and A. Kusenko, Self-interacting asymmetric dark matter coupled to a light massive dark photon, J. Cosmol. Astropart. Phys. 07 (2014) 039.

[35] M. R. Buckley, J. Zavala, F.-Y. Cyr-Racine, K. Sigurdson, and M. Vogelsberger, Scattering, damping, and acoustic oscillations: Simulating the structure of dark matter halos with relativistic force carriers, Phys. Rev. D 90, 043524 (2014).

[36] K. K. Boddy, J. L. Feng, M. Kaplinghat, and T. M. P. Tait, Self-interacting dark matter from a non-Abelian hidden sector, Phys. Rev. D 89, 115017 (2014).

[37] K. Schutz and T. R. Slatyer, Self-scattering for dark matter with an excited state, J. Cosmol. Astropart. Phys. 01 (2015) 021

[38] J. F. Navarro, C. S. Frenk, and S. D. White, A universal density profile from hierarchical clustering, Astrophys. J. 490, 493 (1997).

[39] B. Moore, Evidence against dissipationless dark matter from observations of galaxy haloes, Nature (London) 370, 629 (1994).

[40] R. A. Flores and J. R. Primack, Observational and theoretical constraints on singular dark matter halos, Astrophys. J. 427, L1 (1994).

[41] A. A. Klypin, A. V. Kravtsov, O. Valenzuela, and F. Prada, Where are the missing galactic satellites?, Astrophys. J. 522, 82 (1999).

[42] B. Moore, S. Ghigna, F. Governato, G. Lake, T. Quinn, J. Stadel, and P. Tozzi, Dark matter substructure within galactic halos, Astrophys. J. 524, L19 (1999).

[43] G. Kauffmann, S. D. White, and B. Guiderdoni, The formation and evolution of galaxies within merging dark matter haloes, Mon. Not. R. Astron. Soc. 264, 201 (1993).

[44] M. Boylan-Kolchin, J. S. Bullock, and M. Kaplinghat, Too big to fail? The puzzling darkness of massive Milky Way subhaloes, Mon. Not. R. Astron. Soc. 415, L40 (2011).

[45] L. Liu, B. F. Gerke, R. H. Wechsler, P. S. Behroozi, and M. T. Busha, How common are the Magellanic clouds?, Astrophys. J. 733, 62 (2011).

[46] E. J. Tollerud, M. Boylan-Kolchin, E. J. Barton, J. S. Bullock, and C. Q. Trinh, Small-scale structure in the SDSS and LCDM: Isolated $\sim L *$ galaxies with bright satellites, Astrophys. J. 738, 102 (2011).

[47] L. E. Strigari and R. H. Wechsler, The cosmic abundance of classical Milky Way satellites, Astrophys. J. 749, 75 (2012).

[48] Se-Heon Oh, C. Brook, F. Governato, E. Brinks, L. Mayer, W. J. G. de Blok, A. Brooks, and F. Walter, The central slope of dark matter cores in dwarf galaxies: Simulations vs THINGS, Astron. J. 142, 24 (2011).

[49] C. B. Brook, G. Stinson, B. K. Gibson, R. Roskar, J. Wadsley, and T. Quinn, Hierarchical formation of bulgeless galaxies II: Redistribution of angular momentum via 
galactic fountains, Mon. Not. R. Astron. Soc. 419, 771 (2012).

[50] A. Pontzen and F. Governato, How supernova feedback turns dark matter cusps into cores, Mon. Not. R. Astron. Soc. 421, 3464 (2012).

[51] F. Governato, A. Zolotov, A. Pontzen, C. Christensen, S. H. Oh, A. M. Brooks, T. Quinn, S. Shen, and J. Wadsley, Cuspy no more: How outflows affect the central dark matter and baryon distribution in Lambda CDM galaxies, Mon. Not. R. Astron. Soc. 422, 1231 (2012).

[52] B. Patt and F. Wilczek, Higgs-field portal into hidden sectors, arXiv:hep-ph/0605188.

[53] S. Andreas, T. Hambye, and M. H. Tytgat, WIMP dark matter, Higgs exchange and DAMA, J. Cosmol. Astropart. Phys. 10 (2008) 034.

[54] S. Andreas, C. Arina, T. Hambye, F.-S. Ling, and M. H. Tytgat, A light scalar WIMP through the Higgs portal and CoGeNT, Phys. Rev. D 82, 043522 (2010).

[55] A. Djouadi, O. Lebedev, Y. Mambrini, and J. Quevillon, Implications of LHC searches for Higgs-portal dark matter, Phys. Lett. B 709, 65 (2012).

[56] M. Pospelov and A. Ritz, Higgs decays to dark matter: Beyond the minimal model, Phys. Rev. D 84, 113001 (2011).

[57] A. Greljo, J. Julio, J. F. Kamenik, C. Smith, and J. Zupan, Constraining Higgs mediated dark matter interactions, J. High Energy Phys. 11 (2013) 190.

[58] B. Bhattacherjee, S. Matsumoto, S. Mukhopadhyay, and M. M. Nojiri, Phenomenology of light fermionic asymmetric dark matter, J. High Energy Phys. 10 (2013) 032.

[59] C. Kouvaris, I. M. Shoemaker, and K. Tuominen, Selfinteracting dark matter through the Higgs portal, Phys. Rev. D 91, 043519 (2015).

[60] CMS Collaboration, Precise determination of the mass of the Higgs boson and studies of the compatibility of its couplings with the standard model, Report No. CMS-PASHIG-14-009, 2014.

[61] G. Aad et al. (ATLAS Collaboration), Measurements of Higgs boson production and couplings in the four-lepton channel in pp collisions at center-of-mass energies of 7 and $8 \mathrm{TeV}$ with the ATLAS detector, Phys. Rev. D 91, 012006 (2015).

[62] G. Aad et al. (ATLAS Collaboration), Observation and measurement of Higgs boson decays to $W W^{*}$ with the ATLAS detector, Phys. Rev. D 92, 012006 (2015).

[63] G. Aad et al. (ATLAS Collaboration), Search for the $b \bar{b}$ decay of the Standard Model Higgs boson in associated $(W / Z) H$ production with the ATLAS detector, J. High Energy Phys. 01 (2015) 069.

[64] ATLAS Collaboration, Evidence for Higgs boson Yukawa couplings in the $H \rightarrow \tau \tau$ decay mode with the ATLAS detector, Reports No. ATLAS-CONF-2014-061 and No. ATLAS-COM-CONF-2014-080, 2014.

[65] G. Aad et al. (ATLAS Collaboration), Measurement of Higgs boson production in the diphoton decay channel in pp collisions at center-of-mass energies of 7 and $8 \mathrm{TeV}$ with the ATLAS detector, Phys. Rev. D 90, 112015 (2014).

[66] T. Aaltonen et al. (CDF and D0 Collaborations), Higgs boson studies at the Tevatron, Phys. Rev. D 88, 052014 (2013).
[67] M.E. Peskin and T. Takeuchi, A New Constraint on a Strongly Interacting Higgs Sector, Phys. Rev. Lett. 65, 964 (1990).

[68] K. Olive et al. (Particle Data Group Collaboration), Review of particle physics, Chin. Phys. C 38, 090001 (2014).

[69] M. Shaposhnikov and C. Wetterich, asymptotic safety of gravity and the Higgs boson mass, Phys. Lett. B 683, 196 (2010).

[70] M. Herranen, T. Markkanen, S. Nurmi, and A. Rajantie, Spacetime Curvature and the Higgs Stability During Inflation, Phys. Rev. Lett. 113, 211102 (2014).

[71] K. Kainulainen, K. Tuominen, and J. Virkajärvi, A model for dark matter, naturalness and a complete gauge unification, J. Cosmol. Astropart. Phys. 07 (2015) 034.

[72] D. Akerib et al. (LUX Collaboration), First Results from the LUX Dark Matter Experiment at the Sanford Underground Research Facility, Phys. Rev. Lett. 112, 091303 (2014).

[73] R. Agnese et al. (SuperCDMS Collaboration), Search for Low-Mass WIMPs with SuperCDMS, Phys. Rev. Lett. 112, 241302 (2014).

[74] J. F. Gunion, H. E. Haber, G. L. Kane, and S. Dawson, The Higgs hunter's guide, Front. Phys. 80, 1 (2000).

[75] S. Dittmaier et al. (LHC Higgs Cross Section Working Group), Handbook of LHC Higgs cross sections: 1. Inclusive observables, arXiv:1101.0593.

[76] J. D. Clarke, R. Foot, and R. R. Volkas, Phenomenology of a very light scalar $\left(100 \mathrm{MeV}<m_{h}<10 \mathrm{GeV}\right)$ mixing with the SM Higgs, J. High Energy Phys. 02 (2014) 123.

[77] Y. Zel'dovich, L. Okun, and S. Pikelner, Quarks: Astrophysical and physicochemical aspects, Sov. Phys. Usp. 8, 702 (1966).

[78] B. W. Lee and S. Weinberg, Cosmological Lower Bound on Heavy Neutrino Masses, Phys. Rev. Lett. 39, 165 (1977).

[79] P. Gondolo and G. Gelmini, Cosmic abundances of stable particles: Improved analysis, Nucl. Phys. B360, 145 (1991).

[80] M. G. Walker, M. Mateo, E. W. Olszewski, O. Y. Gnedin, X. Wang, B. Sen, and M. Woodroofe, Velocity dispersion profiles of seven dwarf spheroidal galaxies, Astrophys. J. 667, L53 (2007).

[81] Y. Zhang, Long-lived light mediator to dark matter and primordial small scale spectrum, J. Cosmol. Astropart. Phys. 05 (2015) 008.

[82] K. Enqvist, K. Kainulainen, and V. Semikoz, Neutrino annihilation in hot plasma, Nucl. Phys. B374, 392 (1992).

[83] B. D. Fields, K. Kainulainen, and K. A. Olive, Nucleosynthesis limits on the mass of longlived tau-neutrinos and muon-neutrinos, Astropart. Phys. 6, 169 (1997).

[84] K. Enqvist, K. Kainulainen, and M. J. Thomson, Stringent cosmological bounds on inert neutrino mixing, Nucl. Phys. B373, 498 (1992).

[85] M. Kawasaki, K. Kohri, and N. Sugiyama, MeV scale reheating temperature and thermalization of neutrino background, Phys. Rev. D 62, 023506 (2000).

[86] E. Aprile (XENON1T Collaboration), The XENON1T Dark Matter Search Experiment, Springer Proc. Phys. 148, 93 (2013). 